\title{
Study on Improved Flexible Neural tree Optimization Algorithm
}

\author{
Yu Wang \\ Jilin Business and Technology College \\ 130062, Changchun, China \\ E-mail:wang_zfy@sina.com
}

\begin{abstract}
The BP neural network is easy to fall into local minimum point, the algorithm convergence speed slow, this paper puts forward an improved algorithm of flexible neural tree, introduced the basic theory knowledge of Flexible neural tree, analyzes the characteristics and advantages of the neural tree. The structure optimization and parameter optimization are adopted some optimization algorithm, Introduced the multi expression programming algorithm for optimization of flexible neural tree structure and by using the improved particle swarm algorithm to optimize the parameters of flexible neural tree, Finally the establishment of complete flexible neural tree model.
\end{abstract}

Keywords- flexible neural tree; fitness; Parameter optimization; multi expression programming optimization.

\section{FLEXIBLE NEURAL TREE}

Flexible neural tree (FNT) is a kind of similar to the artificial neural network of evolutionary computation model, with tree structure, FNT is mainly used to solve various classification and prediction problems, the network has two main parts:(1) tree structure; (2) a group of the arithmetic sign. Flexible neural tree is a new type of neural network, it has the advantages of other conventional neural network: (1) the network layer and layer connection is not completely connected, to allow the connection between cross layer; (2) the network intermediate layer and output layer of nonlinear neurons can have not the same or variable as the incentive function ; (3) in the operation process of the neural tree has the change according to the environment to change their lower input space dimension feature; (4) as can change the structure, so the network has the flexibility of flexible structure, and can automatically select problem the input feature [1] .

\section{FleXible Neural Tree Structure Codes}

Flexible neural tree model is composed of instruction set $\mathrm{S}$, the instruction set is divided into two types: non terminal instruction set $\mathrm{F}$ (also known as: function instruction set ) and terminal instruction set $\mathrm{T}$. Non terminal instruction set $\mathrm{F}$ for the tree structure of non-leaf node connected to the sub tree, terminal command is input. The neural tree instruction set is defined as: $S=F \cup T=\left\{+_{2},+_{3}, \ldots,+_{n}\right\} \bigcup\left\{x_{1}, x_{2}, \ldots, x_{n}\right\}$, Which $+_{i}(i=2,3, \ldots, n)$ expresses the non-leaf node instruction with $i$ parameters, and the node and its child nodes between $i$ connection weights. $x_{1}, x_{2}, \ldots, x_{n}$ is leaf node instruction ( input endpoint ). Non - leaf nodes of the output is the calculation result of the flexible neurons, as shown in figure 1 .

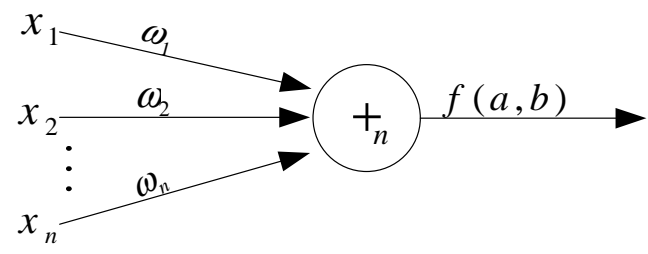

Figure 1. A flexible neuron

In the process of structural neural tree, if a non - leaf node command is selected, For instance $+_{i}$ is selected, it will be randomly generated $i$ non - leaf node and $i$ node and sub tree between the connection weights, and also can produce two random number $\alpha_{i}$ and $b_{i}$ node as the incentive function of parameters. Network model of non - leaf of the incentive function with the following expression:

$$
\left.f\left(a_{i}, b_{i} ; x\right)=\exp \left(\frac{x-a_{i}}{b_{i}}\right)^{2}\right)
$$

Flexible neurons $+_{i}$ output is calculated by the following equation:

$$
\text { net }_{i}=\sum_{j=1}^{i} \omega_{j} * x_{j}
$$

Among them, the flexible neuronal input, then the node total excitation function output for:

$$
\text { out }=f\left(a_{i}, b_{i}, n e t\right)=e^{-\left(\frac{n e_{i}-a_{i}}{b_{i}}\right)^{2}}
$$

\section{FITNESS FUNCTION}

Fitness is reflect a function or a structure quality measure, neural tree model of fitness function represents the actual output and the expected output error, the size of Fitness reflects the neural tree model is good or bad. Adapt to the smaller value that is between the actual output and the 
expected output error is smaller, that is to say to neural tree fitness as small as possible. In this paper, the fitness function is using standard variance calculation. Standard deviation formula:

$$
\operatorname{Fit}(\mathrm{i})=\sqrt{\frac{1}{\mathrm{n}} \sum\left(y_{1}^{j}-y_{2}^{j}\right)^{2}}
$$

Here $n$ says the total amount of sample, $y_{1}^{j}$ and $y_{2}^{j}$ said the $\mathrm{j}$ sample of the actual value and the actual output of the neural tree value, the Fit (i) said $i$ neural tree to value [2].

\section{Multi Expression Programming Optimization OF FLEXIBLE NEURAL TREE}

Multi expression programming (MEP) is by Romania scholar Oltean.M and Dumitrescu. D. in 2002 raised a new linear coding genetic algorithm [3,4]. Multi expression programming algorithm is a novel genetic algorithm. With other linear coding genetic algorithm to compare, multi expression programming features a chromosome contains a plurality of expressions, Each of these expressions corresponding to one of the problem as a solution, namely each expression are likely to represent the chromosome. Therefore, MEP of one chromosome contains multiple solutions to problems. Multi expression programming is a new evolutionary algorithm; this algorithm inherits the evolution steps of genetic algorithm. Including the crossover operator and mutation operator, as shown is in table 1 and table 2.

TABLE I. INTRODUCTION CROSSOVER OPERATOR

\begin{tabular}{|l|l|}
\hline \multicolumn{1}{|c|}{$\begin{array}{c}\text { Crossover } \\
\text { operator }\end{array}$} & \multicolumn{1}{|c|}{ introduction } \\
\hline $\begin{array}{l}\text { Single point } \\
\text { crossover } \\
\text { operator }\end{array}$ & $\begin{array}{l}\text { Random choice of hybridization, chromosome } \\
\text { from hybridization to the chromosome end } \\
\text { exchange; }\end{array}$ \\
\hline $\begin{array}{l}\text { Two point } \\
\text { crossover } \\
\text { operator }\end{array}$ & $\begin{array}{l}\text { Random selection two hybridization in } \\
\text { chromosome, chromosome exchange between } \\
\text { two points; }\end{array}$ \\
\hline $\begin{array}{l}\text { Multi point } \\
\text { crossover } \\
\text { operator }\end{array}$ & $\begin{array}{l}\text { Random selection of several hybrid point to } \\
\text { gene exchange. }\end{array}$ \\
\hline
\end{tabular}

TABLE II. INTRODUCTION MUTATION OPERATOR

\begin{tabular}{|l|l|}
\hline $\begin{array}{c}\text { Mutation } \\
\text { operator }\end{array}$ & \multicolumn{1}{|c|}{ introduction } \\
\hline $\begin{array}{l}\text { Standard } \\
\text { variation } \\
\text { operator }\end{array}$ & $\begin{array}{l}\text { In the standard mutation operator, random } \\
\text { selection of genes in chromosome as a mutation } \\
\text { operation object; }\end{array}$ \\
\hline
\end{tabular}

\begin{tabular}{|l|l|}
\hline $\begin{array}{l}\text { Uniform } \\
\text { variation } \\
\text { operator }\end{array}$ & $\begin{array}{l}\text { In the uniform mutation, in chromosome all } \\
\text { genes in probability P mutation . And the } \\
\text { standard variation, uniform mutation can expand } \\
\text { to search the solution space. }\end{array}$ \\
\hline
\end{tabular}

We use multi expression programming to build the neural tree structure, suppose function set $F=\left\{+_{2},+_{3},+_{4},+_{5},+_{6}\right\} \quad ; \quad$ end point set $T=\left\{x_{1}, x_{2}, x_{3}\right\}$. The said one of the chromosome as shown in figure 2, If the gene 8 Fitness best, choose gene 8 to represent the whole chromosome is shown in figure 3 . With the parameters of multi expression programming representation neural tree model is shown in table 3.
1. $\mathrm{x} 2$

2. $\mathrm{x} 3$

3. $+2,1,2$

4. $\mathrm{x} 1$

5. $+3,1,2,4$

6. $+3,4,1,2$

7. $+2,5,4$

8. $+4,3,2,6,7$

9. $+6,1,2,4,6,7,8$

Figure 2. Neural Tree chromosome no parameters

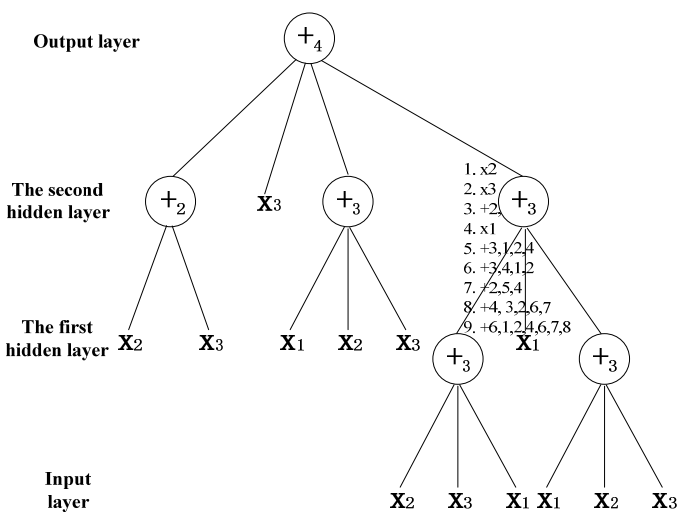

Figure 3 . the 8 genes expressed in neural tree

TABLE III. PARAMETERS OF THE NEURAL TREE WITH A CHROMOSOME

\begin{tabular}{|c|c|c|c|c|c|c|c|c|c|c|c|}
\hline 1 & $\mathrm{x}_{2}$ & & & & & & & & & & \\
\hline 2 & $\mathrm{x}_{3}$ & & & & & & & & & & \\
\hline 3 & $+_{2}$ & 1 & 2 & & & 0.150 & 0.867 & & & 0.294 & 0.223 \\
\hline 4 & $\mathrm{x}_{1}$ & & & & & & & & & & \\
\hline 5 & $+_{3}$ & 1 & 2 & 4 & & 0.528 & 0.639 & 0.941 & & 0.568 & 0.254 \\
\hline
\end{tabular}




\begin{tabular}{|l|l|l|l|l|l|l|l|l|l|l|l|}
\hline 6 & $++_{3}$ & 4 & 1 & 2 & & 0.235 & 0.242 & 0.564 & & 0.255 & 0.956 \\
\hline 7 & $+_{3}$ & 5 & 4 & 6 & & 0.358 & 0.882 & 0.924 & & 0.358 & 0.654 \\
\hline 8 & +4 & 3 & 2 & 6 & 7 & 0.782 & 0.254 & 0.952 & 0.687 & 0.824 & 0.978 \\
\hline
\end{tabular}

\section{Parameter Optimization of FLeXible NeUral TREE}

Flexible neural tree related parameter optimization in this paper by using improved particle swarm optimization algorithm. Particle swarm optimization algorithm (PSO) is a kind of evolutionary computation techniques, with genetic algorithm is similar. First is made up of the United States Eberhart and Professor Kennedy inspired by the flock foraging behavior of [5]. Because the PSO algorithm is relatively simple, and easy to understand, so PSO algorithm in recent years has been rapid development. In order to further illustrate the principle process of PSO , the following according to figure 4 shows.

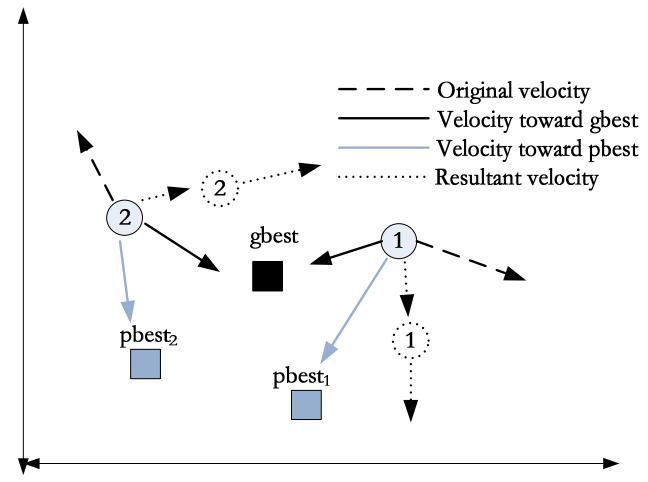

Figure 4. The schematic diagram of the basic PSO algorithm

From the figure 4 more intuitive understanding of the PSO algorithm, which is in a two-dimensional space has two movements of the particles 1 and particles 2, In the previous best position pbest 1 and pbest 2 and the global best position gbest below joint action, make two particles away from the original direction of motion but tend to best position direction of motion. Such particles can escape from local optimal position, and gradually converge to the global optimal location. The PSO algorithm in the research process find the algorithm is easy to fall into local optimum, also can appear premature convergence [6], for these defects once again put forward some new improved algorithm. In this paper based on change the dynamic inertia weight , put forward a kind of regional selection particle swarm optimization ( RSPSO ). According to the different positions of each particle is divided into different regions, also said that each particle can represent them, can also represent its area. The particle $X=\left\{x_{1}, x_{2}, \cdots \cdots, x_{n}\right\}$, the particle as a center, extending outwardly the same distance $R$. Thus obtained to particle $X$ as center, take $R$ as the radius of the region of space, because the two different particle Euclidean distance may be less than the radius $R$, so we allow the overlapping region, According to this method, if the number of groups for the $m$, can put the global area partition of $m$ different sub region. As shown in Figure 5, shows 3 particle regions. Where $n$ represents dimension, $K$ represents the number of particles.

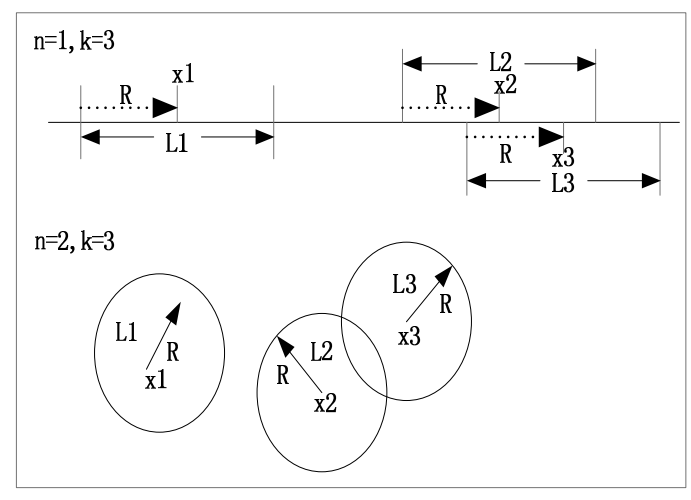

Figure 5. The particle area

If $n=1$, area is one-dimensional and each region is a line segment; if $n=2$, area is two-dimensional and each region is a circle. Each individual represents a subset of region, and the region is the current optimal solution, namely Pbest. In order to find the optimal solution of the area, each particle within the region of evolution and selection of regional optimal solution, evolution rules are as follows:

If $X=\left\{x_{1}, x_{2}, \cdots \cdots, x_{n}\right\}, x_{i}$ is vector $\mathrm{i}$ dimension, new generation $X^{\prime}=\left\{x_{1}^{\prime}, x_{2}^{\prime}, \cdots \cdots, x_{n}^{\prime}\right\}$ is as follows:

$$
x_{i}^{\prime}=N\left(x_{i}, R\right)
$$

Among them $N\left(x_{i}, R\right)$ is Gauss distribution function, if $x_{i}^{\prime}$ beyond the regional scope, to evolve, so in order to limit the area.

RSPSO algorithm steps are as follows:

Step 1: initializing particle population dimensions, particle size (number of individuals), speed, number of iterations and other parameters;

Step 2: according to the particle size division the space , each particle represents a space, evolution to generate the next generation;

Step 3: Calculation of each particle progeny and parent adaptive value, according to the size of adaptive value judgment to leave father generation or offspring;

Step 4: comparison of previous best position corresponding to the value and the current value of the objective function. If the current value is better, it is with the current xid (current particle location) to replace pid (individual optimal particle position); Otherwise, the pid remain unchanged; To determine the optimal value of gbest 
(global optimal particle location). It was finished update particle of the global optimal value and optimal value;

Step 5: check whether meet the PSO algorithm termination conditions, if not, turn to step 5, the next search; If it is, the output gbest and the corresponding optimal adaptive value;

Step 6: using iterative speed and position formula of particle swarm update, the iteration number add 1 , and to step 2.

\section{Establish the Optimization Process of FleXible NEURAL TREE}

Algorithm is described as follows:

(1) Initialization operation: setting multi expression programming and particle swarm algorithm parameter initial value;

(2) the structure optimization: use more expression programming optimization algorithm to optimize the structure of the neural tree model, including adaptive value function to use standard deviation calculated; And also measured by fitness structure, choose the best structure.

(3) If find a suitable structure of flexible neural tree, jumped to the fourth step, or turn to step 2 to structure optimization;

(4) Parameter optimization: use the improved particle swarm algorithm to optimize the parameters of flexible neural tree model, these parameters are mainly the connection weights between the node and the variable parameters of incentive function, The structure of flexible neural tree is fixed, the structure from the results of the step;

(5) If the iteration number of particle swarm optimization has been the biggest, or find a better parameter vector, then jump to step 6.
(6) When find satisfactory results or other termination conditions meet stop the whole algorithm, or turn to step 4;

\section{SUMMARY}

Neural tree is the further improvement of the neural network, so the principle of flexible neural tree more indepth research and analysis, the optimization of the structure and parameter optimization are adopted some optimization algorithm, The structure we adopted more expression programming optimization; The parameters we used the improved particle swarm algorithm optimization. Such training out of the flexible neural tree more plays a classifier role, as the last stage of the classification plays the key role. That is to say the stand or fall of classifier also determines the accuracy of the classification results.

\section{REFERENCES}

[1] J.W. Zhao, G.F. Jia, Q.T. Hou, Y.H. Chen. "analog circuit fault diagnosis methods Based on the flexible immune neural tree ",Journal of shandong science, 2009, 22 (1) : pp. 35- 39.

[2] P. Wu, Z.Liu, Y.F. Chen. "Time series forecasting based on neural tree ", Shandong science, 2007,20 ( 1): pp. 59-64.

[3] Oltean M,Dumitrescu D.Multi “expression programming”,.ClujNapoca: Babes-Bolyai University,2002.

[4] Oltean M,Grosan C. "Evolving evolutionary algorithms using multi expression programming”,.Banzhaf W.The 7th European Conference on Artificial Life.Berlin:Springer-Verlag,2003: pp. 651-658.

[5] Kennedy J,Eberhart R. "Particle Swarm Optimization ”, IEEE on Networks, 1995. pp. 1942-1948.

[6] C.R. Wang, X.D. Duan, X.D. Liu. "Improved basic particle swarm optimization algorithm”, Computer Engineering, 2004,30 ( 21): pp. 35-37.. 